\title{
Hole-hole correlations in the $U=\infty$ limit of the Hubbard model and the stability of the Nagaoka state
}

\author{
M.W. Long \\ School of Physics \\ University of Birmingham \\ Edgbaston, Birmingham B15 2TT, United Kingdom \\ X. Zotos \\ Institut Romand de Recherche Numérique en Physique des Materiaux (IRRMA) \\ PHB-Ecublens, CH-1015 Lausanne, Switzerland
}

\begin{abstract}
We use exact diagonalisation in order to study the infinite - $U$ limit of the two dimensional Hubbard model. As well as looking at single-particle correlations, such as $n_{\mathbf{k} \sigma}=\left\langle c_{\mathbf{k} \sigma}^{\dagger} c_{\mathbf{k} \sigma}\right\rangle$, we also study $N$-particle correlation functions which compare the relative positions of all the particles in different models. In particular we study 16 and 18-site clusters and compare the charge correlations in the Hubbard model with those of spinless fermions and hard-core bosons. We find that although low densities of holes favour a 'locally-ferromagnetic' fermionic description, the correlations at larger densities resemble those of pure hard-core bosons surprisingly well .
\end{abstract}

$71.30,74.20,75.10 \mathrm{~J}$

Typeset Using REVTEX 
In the following we will study the hole-hole correlations in the t-model given by the $J \rightarrow 0$ limit case of the Hamiltonian:

$$
H=-t \sum_{\langle i j\rangle \sigma}\left(\tilde{c}_{i \sigma}^{\dagger} \tilde{c}_{j \sigma}+h . c\right)+J \sum_{\langle i j\rangle}\left(\vec{S}_{i} \cdot \vec{S}_{j}-n_{i} n_{j} / 4\right)
$$

where $\tilde{c}_{i \sigma}\left(\tilde{c}_{i \sigma}^{\dagger}\right)$ are annihilation (creation) operators of a fermion on site i with spin $\sigma$ and the tilde implies the restriction to single occupancy. The sum is over all bonds $\langle i j\rangle$ of a two dimensional lattice with periodic boundary conditions.

This Hamiltonian is the generic model for a doped antiferromagnet and also a true Hubbard model in the limit $U=\infty$. We have chosen the case with no spin fluctuations because a finite $J$ induces attraction between the holes forming pairs (for larger values of $J$ even phase separation) and we want to study the simple effect of the existence of a spin background on the motion of the holes. Although it is by no means well established, it seems reasonable that we can interpret the t-model in terms of a competition between two effects: Firstly, we have an interaction which promotes ferromagnetism locally around an isolated hole, an effect resulting from the physics inherent in Nagaoka's Theorem [1], and secondly we have an interaction which promotes low-spin, coming from the desire for a low-density of particles to have a paramagnetic ground state (see Kanamori [2]). We will refer to the saturated ferromagnetic state indicated by the first phenomenon as the 'Nagaoka state'.

We consider a lattice with $\mathrm{N}$ sites, away from half-filling with $N_{c}$ fermions and $N-N_{c} \equiv$ $N_{h}$ empty sites (or holes). In a previous work [3] we presented a variational argument indicating that the Nagaoka state should be unstable and the holes should have correlations and energy close to that of hard core bosons (h.c.b.). The argument is as follows; the ground state wavefunction of the combined hole-spin system can always be factorized into the product of a charge part times a spin part.

$$
\left|\Psi>=\sum_{\{c\}} \alpha_{c}\right| c>\left(\sum_{\left\{s_{c}\right\}} \beta_{s_{c}} \mid s_{c}>\right)
$$

where the first sum is over all charge configurations $\mid c>$ and the second sum is over all spin configurations $\mid s_{c}>$ for the given hole configuration $\mid c>$. Further we have chosen the 
factors $\alpha_{c}$ positive and the coefficients $\beta_{s_{c}}$ such that:

$$
\sum_{\left\{s_{c}\right\}}\left|\beta_{s_{c}}\right|^{2}=1
$$

We can then define a unit vector in spin space $\vec{\Sigma}_{c}=\sum_{\left\{s_{c}\right\}} \beta_{s_{c}} \mid s_{c}>$. One way to implement this description is in terms of a 'slave-fermion' representation: $c_{i \sigma}^{\dagger}=f_{i}^{\dagger} b_{i \sigma}^{\dagger}$ where $f_{i}^{\dagger}$ is a pure fermion operator which carries the statistics and $b_{i \sigma}^{\dagger}$ is a pure boson which carries the spin. In these terms $\left|c>=\prod_{i \epsilon I} f_{i}^{\dagger}\right| 0>$ and $\left|s_{c}>=\prod_{i \epsilon I} b_{i \sigma_{i}}^{\dagger}\right| 0>$, corresponding to the state with electrons on the sites contained in $I$ with spins $\sigma_{i}$. In this representation the ground state energy $E$ can then be written as:

$$
E=<\Psi|\hat{T}| \Psi>=\sum_{\left\{c^{\prime}, c\right\}} \alpha_{c^{\prime}} \alpha_{c}<c^{\prime}|\hat{T}| c>\vec{\Sigma}_{c^{\prime}} \cdot \vec{\Sigma}_{c}
$$

In this expression the charge configurations $|c\rangle, \mid c^{\prime}>$ are related by the hop of a single particle between neighboring sites with amplitude $\left\langle c^{\prime}|\hat{T}| c\right\rangle= \pm t$ because of the fermionic character of the particles. The product in spin space $\vec{\Sigma}_{c^{\prime}} \cdot \vec{\Sigma}_{c}$ can take values between \pm 1 . In the case of the completely ferromagnetic state the spin configurations $\vec{\Sigma}_{c}$ are 'parallel', so the product is always +1 and the energy of the system is that of a Fermi sea filled with same-spin fermions. In principle however the system could minimize its energy by using the spin degrees of freedom which we can consider as variational parameters. In the ideal case whenever the sign of the hopping is + t the 'spin wavefunctions' should be 'antiparallel' thus cancelling the fermionic sign exactly. In this case then the holes would have exactly the same energy and charge correlations (expressed by the $\alpha_{c}$ 's) as free hard core bosons. We should note that as the spin fluctuation term is zero there is no energy associated with spin flips. Actually as we will see this 'fermion' to 'bosons' transformation works perfectly for a system of a single square plaquette because the spin configuration in this case corresponds to a singlet.

To decide about the behavior of holes in the presence of the spin background we studied, besides the energy, the hole-hole correlations. In particular we want to compare the hole-hole correlations in the t-model with the correlations of particles in different reference models, 
such as hard core bosons or spinless fermions. As a measure of the resemblance we defined an 'overlap' between the hole-hole correlations in the t-model and the reference model as:

$$
(t \mid r)=\sum_{i_{1}<i_{2}<, \ldots,<i_{N_{h}}} \sqrt{<\Psi_{t}\left|n_{i_{1}} n_{i_{2}} \ldots n_{i_{N_{h}}}\right| \Psi_{t}>} \cdot \sqrt{<\Psi_{r}\left|n_{i_{1}} n_{i_{2}} \ldots n_{i_{N_{h}}}\right| \Psi_{r}>}
$$

where $n_{i}=1-f_{i}^{\dagger} f_{i}=1-n_{i}^{c}$ is the hole number operator at site $\mathrm{i}$ in the t-model or the particle number in the reference model and $N_{h}$ is the number of holes. $\left|\Psi_{t}\right\rangle$ is the ground state wavefunction of the t-model and $\left|\Psi_{r}\right\rangle$ of the reference model. It is easy to see that this overlap $\leq+1$, taking the value +1 when all the charge correlations are identical. We should also note that the factors $\alpha_{h} \equiv \alpha_{c}=\sqrt{<\Psi_{t}\left|n_{i_{1}} n_{i_{2}} \ldots n_{i_{N_{h}}}\right| \Psi_{t}>}$ as defined above, since $<\Psi_{t}\left|n_{i_{1}} n_{i_{2}} \ldots n_{i_{N_{h}}}\right| \Psi_{t}>=<\Psi_{t}\left|n_{j_{1}}^{c} n_{j_{2}}^{c} \ldots n_{j_{N_{c}}}^{c}\right| \Psi_{t}>$, where $i_{n}$ are the sites of the holes and $j_{n}$ are the complementary sites of the particles. This overlap is a rather global comparison of the hole-hole correlations between the t-model and the reference model and is not extremely sensitive as the differences enter as quadratic effects; viz $\sum_{\{c\}} \tilde{\alpha}_{c} \cdot \alpha_{c}=1-\frac{1}{2} \sum_{\{c\}}\left(\tilde{\alpha}_{c}-\alpha_{c}\right)^{2}$.

We will first present the results for different size systems, from 4 to 18 sites and different hole densities. They were obtained by exact diagonalization of the Hamiltonian using the Lanczos technique. For the present N-particle correlation functions it is by no means clear whether or not Quantum Monte Carlo techniques can evaluate these quantities. As we discussed above, for the system with 4 sites and 2 holes the ground state is a singlet $(S=0)$ and the energy is $E=-2 \sqrt{2}$, the same as that for hard core bosons. The simple explanation of this fact is the observation that for two fermionic holes exchanging in the presence of a singlet, they exchange as hard core bosons, the fermion minus sign cancelled by the rotation of the singlet wavefunction. Another point of view is that the singlet spin wavefunction corresponds to a rotation of the boundary conditions which shifts the allowed $k$ vectors from the set $\{0, \pm \pi / 2, \pi\}$ to $\{ \pm \pi / 4, \pm 3 \pi / 4\}$.

The next system where this transformation from fermion to boson correlations is exact is the 8 site system. In this case one of the ground states is a singlet $(S=0)$ with energy $E=-2 \sqrt{2} \sqrt{3}=-4.8990$. For comparison the energy for 2 hard core bosons in this lattice is $E_{h . c . b}=-4 \sqrt{3}=-6.9282$ and for 2 spinless fermions $E_{f}=-4$. What is interesting 
in this system is that the hole-hole correlations are identical to those of two hard core bosons, overlap ( $\mathrm{t} \mid$ h.c.b) $=+1$, although the energy is much higher (rather closer to that of free spinless fermions -the Nagaoka state-). This observation is, as we show below, true for almost all systems we studied.

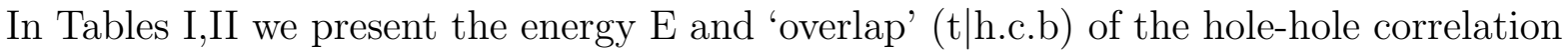
functions to those of hard core bosons as a function of $S^{z}$, the number of spin flips from the totally ferromagnetic state -the Nagaoka state -, for different number of holes $N_{h}$. As we have mentioned above our criterion of the 'overlap' of correlation functions is not sensitive to the details, so on the right hand side of these tables we also give the hole-hole pair correlation function $g(\vec{r})_{t}=<n_{0} n_{r}>$ for different number of holes in the ground state as well as those of hard core bosons $g(\vec{r})_{h . c . b}=<n_{0} n_{r}>$ in the ground state.

We should note that we present results for number of holes where the ground state is a singlet; in these cases the momentum of the ground state is $\vec{k}=(0,0)$ and therefore the wavefunction isotropic, the same as that of the hard core boson system. However for $S^{z} \neq 0$ the lowest energy state can be degenerate with $\vec{k} \neq(0,0)$ corresponding to an anisotropic wavefunction rendering the comparison to hard core bosons more tentative. In general the behavior of the ground state spin for different numbers of holes is rather erratic (for further results see ref. [目, 5]), probably due to competition and finite size effects, as we will discuss below. Interesting is also the case of 16 sites with 5 holes, corresponding to the case of a 'closed shell', where the energy is not a monotonic function of $S^{z}$ initially increasing with the first spin flip and then decreasing, with the ground state at $S=1 / 2$.

Comparison with free spinless fermions gives an overlap for $\mathrm{N}=16$ sites: $(\mathrm{t} \mid \mathrm{s} . \mathrm{f})=0.9211$, 0.8899, 0.8247 for $N_{h}=2,4,6$ respectively, while for $\mathrm{N}=18$ sites: (t|s.f) $=0.9373,0.9129$ for $N_{h}=2,4$. Finally comparing with a Gutzwiller projected wavefunction for 10 fermions in 16 sites (a nondegenerate, $\vec{k}=(0,0)$ ground state) we obtain an overlap of 0.9695 ; so for both a spinless fermion and a Gutzwiller wavefunction we find an overlap significantly smaller than that of hard core bosons.

In an attempt to get an insight into the spin part of the wavefunctions we also calculated 
the Fermi distribution function $n_{\sigma}(k)=\left\langle\tilde{c}_{k \sigma}^{\dagger} \tilde{c}_{k \sigma}>\right.$. The tilde implies the restriction to single occupancy. The results are presented in Tables $\amalg \mathrm{II}, \mathrm{IV}, \mathrm{V}$ (for comparison, we also give the results for the Nagaoka state). We remark from the results for 2 holes that the Fermi distribution is very close to that of the Nagaoka state (ferromagnetic, $S=$ max but in the $S^{z}=0$ subspace) with the 'Fermi surface' at approximately $2 k_{F}$ (as in the Nagaoka state) in distinction with results for finite $J$ where the Fermi vector seems to be at $k_{F}$ [6].

We can summarize the results from the tables above: i) decreasing $S^{z}$ the resemblance of the hole-hole correlations to those of hard core bosons increases; for low hole densities a small number of spins flips from the Nagaoka state suffices to attain the maximum overlap $\simeq 0.99$, while for larger densities more spin flips are necessary, ii) the hole pair correlations functions for higher hole densities are closer to those of hard core bosons than for low densities, iii) the Fermi distribution function $n_{\sigma}(k)$ for low hole densities is close to that of spinless fermions (Nagaoka state), iv) decreasing $S^{z}$ the energy is lowered, remaining though closer to that of spinless fermions (the $S^{z}=\max$ case).

At this point we can discuss our variational argument in light of the numerical results presented: our study of two holes corresponds to the low-density limit, where the 'Nagaoka' effect dominates. The correlations indicate that a fermionic interpretation is natural and our variational argument is unhelpful. When we move to higher hole-densities, viz around $33 \%$, however, the hard-core boson description becomes much more relevant yielding a rather accurate description for the charge motion. The difficulty is in understanding why the description is so successful, when a comparison of the energies is so bad.

Usually in variational calculations, the energies are fairly easy to obtain whereas the wavefunctions are very difficult. The reason for this is simply that errors in the wavefunction lead to quadratic errors in the energy. We do not perform a variational calculation in a technical sense, but are simply using the idea of the spin-system as playing the role of variational parameters in an interpretation of our problem. Perhaps the simplest way of explaining this idea is to think about the Schrödinger equation for our chosen representation: 


$$
E \alpha_{c} \vec{\Sigma}_{c}=\sum_{\left\{c^{\prime}, c\right\}} \alpha_{c^{\prime}}<c^{\prime}|\hat{T}| c>\vec{\Sigma}_{c^{\prime}}
$$

This result is quite general and of little practical analytical value, although we do use it to perform our numerical calculations. Our analytic interpretation comes from the fact that we can 'integrate out' the spin degrees of freedom and obtain an effective 'Hamiltonian' for the charge motion in isolation:

$$
E \alpha_{c}=\sum_{\left\{c^{\prime}, c\right\}} \alpha_{c^{\prime}}\left\langle c^{\prime}|\hat{T}| c>\vec{\Sigma}_{c^{\prime}} \cdot \vec{\Sigma}_{c}\right.
$$

In concept, this equation is quite similar to that for the hard-core boson problem. The particles hop to nearest neighbour sites with a particular matrix element. For the hard-core Bose gas, the matrix element is always $-t$, independent of the configuration of the bosons. For the t-model, the matrix element is $\left\langle c^{\prime}|\hat{T}| c\right\rangle \vec{\Sigma}_{c^{\prime}} \cdot \vec{\Sigma}_{c}$, which ranges theoretically between $+t$ and $-t$. For our representation this matrix element depends on the particular configuration of all the other particles. If this matrix element wildely fluctuated in sign, then our description would be of no practical help, but in practice, although this matrix element is reduced from unity, it does not vary much in magnitude. This fact successfully explains our variational results: The eigenvalue for our system becomes proportional to this matrix element, while the wavefunction remains almost identical to that for hard-core bosons.

It also becomes clear what we mean by using the spin system as 'variational parameters'. The overlaps between the different spin wavefunctions, $\vec{\Sigma}_{c^{\prime}} \cdot \vec{\Sigma}_{c}$, are these variational parameters in practice. They must be chosen so as to keep the phase as uniform as possible, in order to avoid quantum mechanical phase cancellation, and then further to optimise the magnitude of the resulting hopping, subject to the constraint that each spin configuration is overlapped with many others, each with different local requirements.

For the eight site model with two holes these overlaps are exactly uniform (up to the sign necessary to cancel the sign of $\left.\left\langle c^{\prime}|\hat{T}| c\right\rangle\right)$ ), taking the value $\frac{1}{\sqrt{ } 2}$, but simultaneously leading exactly to the hard-core bose ground state. For the larger systems this compensation is not exact, but is an understandable explanation for our results. 
A large amount of work has been devoted to the question of the stability of the Nagaoka state. There are two kind of approaches to this question: one is the study of small clusters, the other the calculation of the energy gain or loss when one spin is flipped from the Nagaoka state. Recent studies [0 9] essentially conclude that the Nagaoka state is stable against a single spin flip. They also point out that the study of small clusters is rather hazardous as most often most hole densities correspond to open shells and therefore it is difficult to obtain good systematics. Opposite conclusion is reached by high temperature expansion [10]. We would think however that the case of closed shells might not be representative as there is an energy gap appearing due to the finite size of the clusters which is not representative of the macroscopic system. This way electron-hole or collective excitations are supressed, thus enhancing the stability of the Nagaoka state. Second, seen from the variational point of view above it seems that there is a large variational freedom due to spin flips and in general the case of a finite density of spin flips should be studied in order to decide about the stability of the Nagaoka state. Of course the variational argument above does not exclude the possibility that the magnetization is finite (even $\sim 1$ ) in the thermodynamic limit; if the Nagaoka state turns out to be stable we should explain why the variational argument does not work.

We can conclude that perhaps the most obvious fact from our small clusters is that the energies and quantum numbers of small system ground-states are erratic and dominated by the particular boundary conditions for the cluster. It is however possible to suggest a few 'trends' which the calculations exhibit. Firstly, the two-hole systems exhibit strong fermionic character. This is evidenced by the strong correspondence between the single- $\left(\operatorname{viz} \mathrm{n}_{\sigma}(\mathrm{k})\right)$ correlations for the t-model and spinless fermion model. All would be straightforward, if the total-spin of our two-hole ground-states were large, but it is not. In fact, as our variational argument would suggest, the total-spin is zero. This fact is quite tricky to interpret, and requires further understanding. When the concentration of holes is increased we find rather different behaviour. Although the particular ground-state spin is erratic, it becomes true that as the total-spin of the lowest-energy state is reduced, the overlap with the hard-core Bose ground-state increases. We can interpret these results in terms of the competition 
between the low-concentration Nagaoka effect and the higher-concentration 'bosonization'. For high spin the particles gain their energy from single-particle motion as fermions, while for low spin they gain their energy from collective motion as hard-core bosons with a reduced probability of hopping controlled by the spin wavefunction. At low concentration of holes the single-particle motion appears more important while at high concentration the collective motion seems more important.

\section{ACKNOWLEDGMENTS}

We would particularly like to thank P. Prelovsek for many useful discussions as well as H. Castella, D. Baeriswyl, J.M.F. Gunn; also the hospitality of I.S.I. in Torino where this work was started. This work was supported by the NSF/British Council grant no. 83BC-033384 and (X.Z) also acknowledges financial support by the Swiss National Science Foundation under Grant No. 20-30272.90 and the University of Fribourg. 


\section{REFERENCES}

$1 \quad$ Y. Nagaoka, Phys. Rev. 147, 392 (1966).

2 J. Kanamori, Prog. Theor. Phys. 30, 275 (1963).

3 M. Long, X. Zotos, Phys. Rev. B45, 9932 (1992).

4 J.A. Riera and A.P. Young, Phys. Rev.B40, 5285 (1989).

5 H. Fehske, V. Waas,H. Röder and H. Büttner, Phys. Rev. B44, 8473 (1991).

$6 \quad$ W. Stephan, P. Horsch, Phys. Rev. Lett. 66, 2258 (1991).

$7 \quad$ A. Barbieri, J.A. Riera and A.P. Young, Phys. Rev. B41, 11697 (1990).

$8 \quad$ S.A. Trugman, Phys. Rev. B42, 6612 (1990).

$9 \quad$ W. von der Linden and D.M. Edwards, J.Phys. C3, 4917 (1991).

10 W.O. Putikka, M.U. Luchini and M. Ogata, Phys. Rev. Lett. 692288 (1992). 


\section{TABLES}

TABLE I. "Overlaps" (on the left-) and pair correlations functions (on the right hand side respectively) for $\mathrm{N}=16$ sites

\begin{tabular}{|c|c|c|c|c|c|c|c|}
\hline$N_{h}$ & $S^{z}$ & $\vec{k}$ & $\mathrm{E}$ & (t|h.c.b.) & $\vec{r}$ & $g(\vec{r})_{t}$ & $g(\vec{r})_{h . c . b}$ \\
\hline \multirow[t]{8}{*}{2} & 7 & $(\pi / 2,0)$ & -6 . & 0.9098 & $(1,0)$ & 0.0040 & 0.0053 \\
\hline & 6 & $(0,0)$ & -6.2781 & 0.9969 & $(1,1)$ & 0.0078 & 0.0085 \\
\hline & 5 & $(\pi / 2,0)$ & -6.4117 & 0.9858 & $(2,0)$ & 0.0078 & 0.0085 \\
\hline & 4 & $(0,0)$ & -6.5238 & 0.9967 & $(2,1)$ & 0.0117 & 0.0103 \\
\hline & 3 & $(\pi / 2,0)$ & -6.5687 & 0.9892 & $(2,2)$ & 0.0155 & 0.0115 \\
\hline & 2 & $(0,0)$ & -6.6179 & 0.9966 & & & \\
\hline & 1 & $(\pi / 2,0)$ & -6.6430 & 0.9908 & & & \\
\hline & 0 & $(0,0)$ & -6.6775 & 0.9962 & & & \\
\hline h.c.b & & $(0,0)$ & -7.5696 & & & & \\
\hline \multirow[t]{6}{*}{6} & 5 & $(\pi / 2, \pi / 2)$ & -12 . & 0.8955 & $(1,0)$ & 0.0997 & 0.1012 \\
\hline & 4 & $(\pi / 2, \pi / 2)$ & -12.3781 & 0.9595 & $(1,1)$ & 0.1326 & 0.1320 \\
\hline & 3 & $(\pi, \pi / 2)$ & -12.7418 & 0.9854 & $(2,0)$ & 0.1326 & 0.1320 \\
\hline & 2 & $(0,0)$ & -13.2019 & 0.9932 & $(2,1)$ & 0.1352 & 0.1353 \\
\hline & 1 & $(\pi / 2,0)$ & -13.3512 & 0.9955 & $(2,2)$ & 0.1400 & 0.1359 \\
\hline & 0 & $(0,0)$ & -13.7555 & 0.9980 & & & \\
\hline h.c.b & & $(0,0)$ & -16.7881 & & & & \\
\hline \multirow[t]{5}{*}{8} & 4 & $(\pi / 2, \pi / 2)$ & -12 & 0.8611 & $(1,0)$ & 0.2034 & 0.2044 \\
\hline & 3 & $(0,0)$ & -13.1416 & 0.9432 & $(1,1)$ & 0.2477 & 0.2433 \\
\hline & 2 & $(\pi / 2,0)$ & -13.7099 & 0.9787 & $(2,0)$ & 0.2468 & 0.2433 \\
\hline & 1 & $(0,0)$ & -14.2233 & 0.9874 & $(2,1)$ & 0.2402 & 0.2441 \\
\hline & 0 & $(0,0)$ & -14.3475 & 0.9919 & $(2,2)$ & 0.2410 & 0.2456 \\
\hline h.c.b & & $(0,0)$ & -17.9996 & & & & \\
\hline
\end{tabular}


TABLE II. "Overlaps" (on the left-) and pair correlations functions (on the right hand side respectively) for $\mathrm{N}=18$ sites

\begin{tabular}{|c|c|c|c|c|c|c|c|}
\hline$N_{h}$ & $S^{z}$ & $\vec{k}$ & $\mathrm{E}$ & (t|h.c.b.) & $\vec{r}$ & $g(\vec{r})_{t}$ & $g(\vec{r})_{h . c . b}$ \\
\hline \multirow[t]{9}{*}{2} & 8 & $(2 \pi / 3,2 \pi / 3)$ & -6. & 0.9212 & $(1,0)$ & 0.0030 & 0.0042 \\
\hline & 7 & $(2 \pi / 3,0)$ & -6.3160 & 0.9941 & $(1,1)$ & 0.0046 & 0.0064 \\
\hline & 6 & $(0,0)$ & -6.4597 & 0.9946 & $(2,0)$ & 0.0098 & 0.0075 \\
\hline & 5 & $(\pi, \pi)$ & -6.4872 & 0.9932 & $(2,1)$ & 0.0077 & 0.0076 \\
\hline & 4 & $(2 \pi / 3,2 \pi / 3)$ & -6.5095 & 0.9915 & $(3,0)$ & 0.0125 & 0.0083 \\
\hline & 3 & $(\pi, \pi)$ & -6.5451 & 0.9935 & & & \\
\hline & 2 & $(0,0)$ & -6.5727 & 0.9927 & & & \\
\hline & 1 & $(\pi / 3, \pi / 3)$ & -6.5902 & 0.9922 & & & \\
\hline & 0 & $(0,0)$ & -6.6133 & 0.9920 & & & \\
\hline h.c.b & & $(0,0)$ & -7.6212 & & & & \\
\hline \multirow[t]{8}{*}{4} & 7 & $(2 \pi / 3,2 \pi / 3)$ & -10 & 0.9133 & $(1,0)$ & 0.0258 & 0.0278 \\
\hline & 6 & $(0,0)$ & -10.7118 & 0.9714 & $(1,1)$ & 0.0398 & 0.0402 \\
\hline & 5 & $(2 \pi / 3,0)$ & -10.7232 & 0.9901 & $(2,0)$ & 0.0435 & 0.0430 \\
\hline & 4 & $(0,0)$ & -10.8527 & 0.9984 & $(2,1)$ & 0.0461 & 0.0444 \\
\hline & 3 & $(\pi, \pi)$ & -10.9915 & 0.9972 & $(3,0)$ & 0.0460 & 0.0450 \\
\hline & 2 & $(0,0)$ & -11.1321 & 0.9980 & & & \\
\hline & 1 & $(0,0)$ & -11.1468 & 0.9967 & & & \\
\hline & 0 & $(0,0)$ & -11.1742 & 0.9979 & & & \\
\hline h.c.b & & $(0,0)$ & -13.6111 & & & & \\
\hline
\end{tabular}


TABLE III. $n(k)$ for $N=16, N_{h}=2$, (on the right, for Nagaoka state)

\begin{tabular}{cccccccc}
\hline \hline$\pi$ & 0.512 & 0.340 & 0.019 & $\pi$ & 0.500 & 0.375 & 0.000 \\
$\pi / 2$ & 0.509 & 0.513 & 0.340 & $\pi / 2$ & 0.500 & 0.500 & 0.375 \\
0 & 0.513 & 0.509 & 0.512 & 0 & 0.500 & 0.500 & 0.500 \\
$k_{y} / k_{x}$ & 0 & $\pi / 2$ & $\pi$ & $k_{y} / k_{x}$ & 0 & $\pi / 2$ & $\pi$ \\
\hline \hline
\end{tabular}

TABLE IV. $n(k)$ for $N=16, N_{h}=6$, (on the right, for Nagaoka state)

\begin{tabular}{cccccccc}
\hline \hline$\pi$ & 0.212 & 0.096 & 0.066 & $\pi$ & 0.416 & 0.000 & 0.000 \\
$\pi / 2$ & 0.650 & 0.212 & 0.096 & $\pi / 2$ & 0.500 & 0.416 & 0.000 \\
0 & 0.677 & 0.650 & 0.212 & 0 & 0.500 & 0.500 & 0.416 \\
$k_{y} / k_{x}$ & 0 & $\pi / 2$ & $\pi$ & $k_{y} / k_{x}$ & 0 & $\pi / 2$ & $\pi$ \\
\hline \hline
\end{tabular}

TABLE V. $n(k)$ for $N=18, N_{h}=2$, (on the right, for Nagaoka state)

\begin{tabular}{|c|c|c|c|c|c|c|c|c|c|}
\hline$\pi$ & & 0.497 & & 0.039 & $\pi$ & & 0.500 & & 0.000 \\
\hline $2 \pi / 3$ & 0.505 & & 0.344 & & $2 \pi / 3$ & 0.500 & & 0.375 & \\
\hline$\pi / 3$ & & 0.518 & & 0.497 & $\pi / 3$ & & 0.500 & & 0.500 \\
\hline 0 & 0.507 & & 0.505 & & 0 & 0.500 & & 0.500 & \\
\hline$k_{y} / k_{x}$ & 0 & $\pi / 3$ & $2 \pi / 3$ & $\pi$ & $k_{y} / k_{x}$ & 0 & $\pi / 3$ & $2 \pi / 3$ & $\pi$ \\
\hline
\end{tabular}

